\title{
ARTERIAL HYPERTENSION AND AORTIC DISEASES
}

\author{
Cristina M. TIRZIU' ${ }^{1 \otimes}$ \\ ${ }^{1}$ Clinical Emergency Hospital of Bucharest, Romania
}

\author{
Received 12 Nov 2020, Accepted 23 Nov 2020 \\ hitps://doi.org/10.31688/ABMU.2020.55.4.14
}

\begin{abstract}
Arterial hypertension (HTN) is one of the most prevalent diseases all over the word. There are many studies and reviews about the coexistence of HTN with coronary, cerebrovascular and renal diseases. However, sometimes clinicians do not search for aortic disease in hypertensive patients. The coexistence of HTN and various aortic diseases raises peculiar issues regarding monitoring and managing of these patients. This review presents data about HTN and aortic aneurism, aortic coarctation and aortic valve disease, emphasizing the particularities of the diagnosis and management of HTN in these patients. Also, a part of the review will focus on the evaluation of arterial stiffness and its clinical implications.
\end{abstract}

Keywords: arterial hypertension, arterial stiffness, aortic aneurism, aortic stenosis, aortic regurgitation.

\section{List of abbreviations \\ HTN - hypertension \\ PWV - pulse wave velocity \\ PP - pulse pressure}

ABPM - ambulatory blood pressure measurement

MAP - mean arterial pressure

AOC - aortic coarctation

RAAS - renin-angiotensin-aldosterone system

AS - aortic stenosis

\section{Résumé}

L'hypertension artérielle et les maladies aortiques

L'hypertension artérielle (HTA) est l'une des maladies les plus répandues dans le monde. Il existe de nombreuses études et revues sur la coexistence de la HTA avec les maladies coronariennes, cérébrovasculaires et rénales. Cependant, les cliniciens ne recherchent parfois pas une maladie aortique chez les patients hypertendus. La coexistence de la HTA et de diverses maladies aortiques pose des problèmes particuliers de suivi et de prise en charge de ces patients. Cette revue présente des données sur la HTA et l'anévrisme aortique, la coarctation aortique et la maladie de la valve aortique, en insistant sur les particularités du diagnostic et de la prise en charge de la HTA chez ces patients. En outre, une partie de la revue se concentrera sur l'évaluation de la rigidité artérielle et ses implications cliniques.

Mots-clés: hypertension artérielle, rigidité artérielle, anévrisme aortique, sténose aortique, régurgitation aortique. 


\section{Background}

Cardiovascular diseases remain a major health problem on a global scale, accounting for the majority (i.e. $60 \%$ ) of deaths in Romania ${ }^{1}$. Of these, arterial hypertension (HTN) is the most common, with an estimated prevalence among the adult population of 45.1\% according to the SEPHAR III study ${ }^{1}$.

The association of HTN with aortic diseases raises several issues regarding its evaluation and treatment. This article aims to review the main aortic diseases that are associated with HTN, between which there often is, albeit not mandatory, a cause-effect relationship.

Aortic diseases are included in the larger spectrum of arterial diseases and can be briefly classified according to etiology (Table 1).

\section{ARTERIAL STIFFNESS AND ARTERIAL HYPERTEN- SION - A BIDIRECTIONAL RELATIONSHIP}

Increased aortic rigidity (arterial stiffness) is one of the earliest negative structural and functional maladaptation, which is diagnosed by the clinical use of a surogate marker, respectively pulse wave velocity (PWV) and the augmentation index. PWV can be estimated by reporting the distance that the pulse wave travels to the time needed to travel from one point (carotid) to another (femural). A PWV greater than $10 \mathrm{~m} / \mathrm{s}$ is considered to indicate a significant alteration of the aortic function in hypertensive patients; the European Society of Cardiology/European Society of Hypertension consider the PWV the gold standard to estimate the arterial stiffness due to its simplicity, accuracy and reproductibility ${ }^{2}$. The additive value of PWV above and beyond traditional risk factors, including SCORE and the Framingham risk score, has been suggested by several studies ${ }^{3}$. However, routine use of PWV measurement is not recommended for daily practice.

Framingham Heart Study investigators have shown that the prevalence of carotid-femoral PWV greater than $12 \mathrm{~m} / \mathrm{s}$ increases from a few percentages before the age of 50 years-old to over $60 \%$ after 70 years of age and elevated PWV is associated with high-risk for incident hypertension ${ }^{4}$.

Pulse pressure (PP), calculated by systolic minus diastolic blood pressure from a conventional blood pressure measurement, is another marker of aortic stiffness that is widely available but generally ignored when stratifying the risk. PP is sensitive to stiffness of the aortic wall and the balance between aortic flow and diameter in the proximal aorta. However, the relationship between PP, which is derived from blood pressure, and carotid-femoral PWV, the current standard indicator of aortic wall stiffness, is complex and potentially divergent, leading to ambiguity and disagreement regarding the role that arterial stiffness plays as a precursor versus complication of hypertension ${ }^{5}$.

Arterial stiffness is the pathophysiological link to isolated systolic HTN. However, there are several experimental and clinical studies demonstrating that injuries that increase the aortic stiffness directly, by disrupting elastin in the aortic wall, are associated with subsequent development of HTN. Considering all these, there are still many questions to be answered about the fact that arterial stiffness is a cause or rather a consequence of HTN.

One interesting observation is that the majority of resistant or difficult to treat HTNs are associated with high arterial stiffness. This observation could have significant clinical implications. Some drugs, such as angiotensin-converting enzyme inhibitors, angiotensin receptor blockers, and low-dose diuretics, have coincidental favorable effects on arterial stiffness $^{6,7}$. In clinical practice, the ambulatory blood pressure measurement (ABPM) is often used, especially in difficult to treat cases, to verify the result of treatment. Sometimes, the mean arterial pressure (MAP) is normal or near normal, but the systolic pressure is still elevated. If we consider only the MAP and try to further reduce it by using arterial

Table 1. The etiological spectrum of aortic diseases.

\begin{tabular}{cccc}
\hline Atherosclerotic disease & Non-atherosclerotic disease & Infectious/inflammatory disease & Miscellaneous diseases \\
\hline Aneurysm & Cystic medial necrosis & Takayasu arteritis & Direct trauma \\
\hline Pseudoaneurysm & Aneurysm & Giant cell arteritis & Intraluminal disease \\
\hline Penetrating ulcer & Aortic dissection & Endocarditis & Poststenotic dilatation \\
\hline Intramural hematoma & Intramural hematoma & Congenital- coarctation \\
\hline Dissection & Annuloaortic ectasis & $\begin{array}{c}\text { Genetic - Marfan's syn- } \\
\text { drome }\end{array}$ \\
\hline $\begin{array}{c}\text { Atheroembolic disease } \\
\text { Rupture of atheromatous } \\
\text { plaques }\end{array}$ & & Arterial hypertension \\
\hline
\end{tabular}


vasodilators, we can be in the situation in which the MAP is normal, the diastolic pressure is potentially dangerous low and the systolic pressure is still uncontrolled. All these clinical considerations should make the clinicians aware about the role of large vessels rigidity and shift the attention from arteriolar resistance alone to the contribution of aortic stiffness in HTN pathophysiology.

\section{Aortic aneurysm}

An aortic aneurysm is any permanent increase in the diameter of the aorta by more than $50 \%$ compared to the normal (i.e. age and sex-adjusted) size of the aorta. From a pathological viewpoint, an aneurysm involves all three layers of the arterial wall. The term "pseudoaneurysm" defines the perivascular accumulation of blood secondary to leakage through a gap in the arterial wall, most often involving the tunica media, with its contents generally being limited to this level by the adventitia or the perivascular fibrous tissue.

Not infrequently, the primary focus of HTN end-organ damage was mostly centered on cardiac, renal, and cerebral complications; nonetheless, the diagnostic and therapeutic impact of HTN on arterial aneurysms should not be underestimated. The prevalence of HTN among patients with an aortic aneurysm is estimated to be about $60 \%{ }^{8}$. Furthermore, the prevalence of arterial HTN among patients with aortic dissection is even greater (approximately $75 \%)^{9}$. If in recent years the association between the two diseases has been increasingly discussed, the data involving HTN as a pathogenic factor in the formation of an aortic aneurysm and its progression or rupture are still incompletely understood. Despite this, it is well known that an effective control of HTN plays an important role in reducing the rupture rate of aneurysms.

\section{Pathogenic issues regarding the correlation between HTN and the aortic aneurysm}

Because most aneurysms are related to atherosclerotic aortic disease, the diagnostic efforts should be primarily focused on its diagnosis. Primarily because of aging, but also of the prolonged action of various cardiovascular risk factors (HTN, smoking, dyslipidemia, diabetes) on the elastic tissue of the arteries, two types of intertwined changes occur simultaneously: accelerated atherosclerosis (often accompanied by calcification) and arteriosclerosis, the combined effects of which results in thickening of the media and increased arterial wall stiffness. The atherosclerotic process is characterized by sequential development, with cell migration and an increase in endothelial permeability to lipoproteins, resulting in their subendothelial deposition, while shear stress caused by HTN accelerates the formation and growth of the atheromatous plaque. An inadequate vascular remodeling thus occurs. The process of atherosclerosis, accelerated by the appearance of HTN, is the pathophysiological basis of atherothrombotic complications and the development of arterial aneurysms. The accelerating effect of HTN on the development of atherosclerosis has been repeatedly observed in in-vivo studies on hyperlipidemic animals; thus, it acts in a twofold manner, by increasing the parietal stress, inducing endothelial dysfunction and local inflammatory processes, as well as by applying excessive shear stress on lipid particles.

Another pathophysiological aspect probably involved in the relationship between HTN and arteriosclerosis is related to hemodynamic factors. The process of arteriosclerosis, accelerated in the presence of HTN, is the substrate of arterial stiffening in the large arteries. The process is characterized by thinning and fragmentation of parietal elastin fibres, with an increase in the capacity to fix calcium ions and an increase in type I collagen synthesis. An increased vascular resistance results in increased systolic blood pressure, with a direct increasing of parietal stress, that often occurs on an arterial wall already affected by atheromatous lesions. Because the arterial pulse wave is reflected as it travels distally and increases the primary pulse wave, the pressure is higher at the level of abdominal aorta than at the thoracic aorta, which could account for an almost 6 -fold increase in the frequency of abdominal rather than thoracic aortic aneurysms ${ }^{8}$. Regarding this aspect, it is interesting to note that dilation of the aortic root is a common clinical finding in hypertensive patients. Valsalva sinus dilation (easily diagnosed by cardiac ultrasound) has been shown to correlate with the involvement of other "target organs" in patients with HTN and especially with cardiac involvement, the latter being expressed by the presence of left ventricular hypertrophy, thus suggesting that ascending aortic dilation is a strong marker of HTN-induced cardiac impairment and should be routinely screened in hypertensive patients.

Furthermore, the possible relationship between arterial aneurysmal disease and renovascular HTN is particularly intriguing. There are several aspects to briefly mention in this regard, namely: renal ischemia secondary to renal artery aneurysms, the presence of a complicated abdominal aneurysm with propagated dissection in the renal arteries, or renal ischemia secondary to embolization from a thrombotic aneurysm.

The natural history of aneurysms is that of progressive growth, the growth rate being influenced by several factors, including HTN. According to Laplace's law, the parietal stress (the main 
determinant of aneurysm progression) is directly correlated with intraluminal pressure. However, there are currently no large-scale studies that accurately reflect the extent to which the existence of HTN alone influences the rate of aneurysm growth. Regarding the risk of rupture of aneurysms, most studies have referred to the correlation between HTN and rupture of saccular intracerebral aneurysms, with little data on the correlation between rupture of aortic or peripheral aneurysms and the existence or severity of HTN, although its involvement in the process is certain.

Another interesting aspect is the impact of HTN on the prognosis of patients who have received surgi$\mathrm{cal} /$ interventional treatment for an aortic aneurysm. Regarding survival after aortic aneurysm surgery, in a retrospective study of 920 patients who have had surgery for abdominal aortic aneurysms, Crawford et $\mathrm{al}^{11}$ found that advanced age, uncontrolled HTN and other cardiovascular comorbidities were present in 95\% of cases with early (at 30 days) post-operative death; regarding long-term prognosis, the presence of uncontrolled HTN had an impact similar to the existence of coronary artery disease. Another important prognostic aspect is the effect of HTN on the reconstructed arterial system, given the fact that the prevalence of HTN was three times higher in patients who developed postoperative pseudoaneurysms than among those in whom this complication was not encountered.

In conclusion, HTN is common in patients with arterial aneurysms and probably plays an important part in their development. Whether the presence of HTN contributes significantly to the acceleration of the growth rate of aneurysms is not entirely clear and requires further evaluation, but there is relevant clinical evidence regarding the involvement of HTN in aneurysms' rupture. After surgical excision/repair of aneurysms, the persistence of uncontrolled HTN negatively influences both the post-operative survival rate and the risk of long-term complications ${ }^{11}$. We can therefore conclude that both arterial aneurysms and arterial dissection should be considered in the same context as cardiac, renal, or cerebral complications of HTN and require aggressive therapy of $\mathrm{HTN}^{12}$.

\section{Management of patients with aortic aneurysms}

Therapeutic approaches to aortic aneurysms include pharmacological, endovascular, and surgical treatment. The pharmacological treatment aims to slow the rate of expansion of the aneurysm, taking also into consideration the associated diseases and risk factors.

As in aortic dissection, beta-blockers are the main class of drugs used to prevent aneurysmal progression (class IIB indication). In addition, HTN and other risk factors (dyslipidemia, diabetes, coronary heart disease) must also be treated; smoking cessation is essential.

Patients with abdominal aneurysms with a diameter between $4-5.4 \mathrm{~cm}$ must be monitored clinically and through imaging (ultrasound or computed tomography) every 6-12 months (class I indication) ${ }^{12}$. Any abdominal aortic aneurysm with a diameter greater than $5.5 \mathrm{~cm}$ requires surgical repair, to lower the risk of rupture (class I indication) ${ }^{12}$. Similarly, in the case of thoraco-abdominal aneurysms, a surgical approach may be favoured when the aortic diameter is greater than $6 \mathrm{~cm}$ (class II B indication) ${ }^{12}$. The thoracic aortic aneurysm has a natural tendency to expand in size, the rate of growth being higher in patients with Marfan's syndrome or other connective tissue disorders. The optimal timing of surgery is less well known than in the case of abdominal aortic aneurysms. The following cut-off values are commonly used when deciding on a surgical approach: aortic diameter $>5.5 \mathrm{~cm}$; in patients with Marfan's syndrome an aortic diameter $>5 \mathrm{~cm}$, and in the case of a bicuspid aortic valve an aortic diameter $>4 \mathrm{~cm}^{12}$.

\section{Aortic dissection}

Aortic dissection is defined by the existence of a longitudinal cleavage plane between the layers of the aortic wall, due to the penetration of blood into the aortic wall through a rupture of the tunica intima, most often as a result of long standing HTN and degenerative changes in the aortic tunica media. The propagation force of the flowing blood determines the centrifugal progression of the dissection front, from the place of intimal rupture to the periphery, creating a "false" vascular lumen inside the aortic wall, separated from the "true" aortic lumen by the tunica intima (intimal fold).

The most common risk factor for aortic dissection is HTN ( $75 \%$ of cases) $)^{9}$; atherosclerosis, a personal history of aortic aneurysm or cardiac surgery or connective tissue diseases are other frequent risk factors. The close correlation between HTN and aortic dissection is certain, the latter being considered a hypertensive emergency. From a chronological point of view, aortic dissection may be described as of acute onset (duration from onset less than 14 days), subacute (15-90 days) and chronic (more than 90 days from onset).

\section{Drug management of aortic dissection}

Management of acute type A aortic dissection involves drug therapy in all forms of aortic dissection and emergency surgical therapy. In acute uncomplicated type B aortic dissection, drug therapy is indicated with an IC recommendation class (with 
imaging monitoring at 3 months), while non-surgical repair therapy has a IIA recommendation class ${ }^{12}$. In the case of complicated acute type B aortic dissection, there is an IC class recommendation for favouring endovascular repair and a IB class recommendation for favouring surgical treatment ${ }^{12}$. In all cases, emergency drug therapy follows the same basic principles (detailed below).

Medical management in these situations has two major objectives: control of blood pressure and heart rate, and pain therapy. The rapid and correct management of HTN in these circumstances is of crucial importance in limiting the progression of the dissection. The current recommendations call for a rapid lowering of the blood pressure (within 20 minutes) to systolic blood pressure values of $<120-100 \mathrm{mmHg}$ and a heart rate of $<60 \mathrm{bpm}^{12}$. The initial medical treatment aims to lower the blood pressure and the systolic ejection force of the left ventricle to prevent the progression of the dissection. The medication of choice are intravenous beta-blockers (propranolol, metoprolol, labetalol). In case of beta-blocker intolerance, short-acting beta-blockers, such as esmolol, or calcium channel blockers may be used. If blood pressure cannot be controlled by beta-blockers, vasodilators with a diminished reflex-tachycardia response can be added (i.e. nicardipine). As the second line of treatment, intravenous nitroglycerin or sodium nitroprusside can be used (for the latter the initial dose is $0.25 \mu \mathrm{g} / \mathrm{Kg} / \mathrm{min}$ and then titrated until the target blood pressure is achieved) (Table 2). To avoid reflex tachycardia and an increase in shear forces, vasodilator medication will be administered only in combination with beta-blockers. Pain therapy involves oxygen therapy and the administration of opioid analgesics.

\section{Aortic coarctation}

Aortic coarctation (AOC) is a localized thickening of the media, which may or may not be associated with hyperplasia of the overlying endothelial tissue. Aortic coarctation is a relatively common congenital pathology, occurring in $5-8 \%$ of cases of congenital heart disorders ${ }^{13}$. It may be an isolated finding or rather, more likely, associated with other abnormalities, most often with a bicuspid aortic valve (approximately two-thirds of patients), patent ductus arteriosus, or a ventricular septal defect.

Classically, the aortic coarctation occurs at the level of the superior thoracic aorta, immediately distal to the origin of the left subclavian artery. Less frequently, stenosis may occur in the distal thoracic aorta or in the abdominal aorta.

The mechanism of occurrence of HTN in patients with AOC is not yet fully understood. The two proposed pathophysiological hypotheses are the mechanical hypothesis and the biological hypothesis. The latter implies the activation of the renin-angiotensin-aldosterone system (RAAS), whereas the former regards the appearance of HTN strictly as a mechanical consequence due to the presence of a fixed obstacle impeding the blood flow. However, this theory does not explain why blood pressure varies widely in different patients for similar degrees of anatomical obstruction, nor why in some situations a certain level of HTN persists after a curative intervention for coarctation. The humoral theory ${ }^{14,15}$ supports the activation of RAAS because of decreased renal perfusion. It should be noted that some studies have failed to demonstrate the existence of elevated serum renin in these patients. Once the patient develops heart failure, a whole new series of humoral factors become involved and will maintain pre-existing pathophysiological changes (vasopressin, natriuretic hormones).

The key factor in the diagnosis of $\mathrm{AOC}$ is the high degree of clinical suspicion of HTN in children, adolescents, or young adults. The typical feature is that of high blood pressure in the upper limbs and normal blood pressure in the lower limbs. Often, the patient is diagnosed due to the consequences of HTN:

Table 2. Pharmacologic therapy of acute aortic dissection.

\begin{tabular}{cc} 
Labetalol (first choice) & $\begin{array}{c}15 \mathrm{mg} \text { i.v. push followed by } 5 \mathrm{mg} / \text { hour i.v. infusion; action onset in } \\
2-5 \mathrm{minutes} \text { and lasts for 2-4 hours }\end{array}$ \\
\hline Esmolol & $\begin{array}{c}\text { Starting dose } 0.5 \mathrm{mg} / \mathrm{Kg} \text { in } 2-5 \mathrm{~min} \text { followed by } 10-20 \mu \mathrm{g} / \\
\mathrm{Kg} / \mathrm{min} \text { i.v. infusion }\end{array}$ \\
\hline Diltiazem & $\begin{array}{c}\text { Starting dose } 0.25 \mathrm{mg} / \mathrm{Kg} \text { push over } 2-5 \mathrm{minutes} \text {, followed by } \\
\text { i.v. infusion at } 5 \mathrm{mg} / \text { hour }\end{array}$ \\
\hline Nicardipine & $\begin{array}{c}\text { Starting dose } 5 \mathrm{mg} / \text { hour with titration every } 5 \mathrm{minutes} \text { by } \\
2.5 \mathrm{mg} / \text { hour up to a maximum i.v. infusion rate of } 15 \mathrm{mg} / \\
\text { hour; long duration of action may be a disadvantage. }\end{array}$ \\
\hline Sodium nitroprusside & $\begin{array}{c}\text { Starting dose } 0.3 \mu \mathrm{Kg} / \mathrm{min} \text {; elimination half-life } \sim 2 \mathrm{~min}- \\
\text { utes; }\end{array}$ \\
\hline Nitroglycerin & $\begin{array}{c}\text { Starting dose } 5 \mu \mathrm{mg} / \mathrm{min} \text { with titration every } 5 \mathrm{~min} \text { up to a } \\
\text { maximum dose of } 200 \mu \mathrm{mg} / \mathrm{min} .\end{array}$ \\
\hline
\end{tabular}


headache, epistaxis in mild forms or hemorrhagic stroke (due to the frequent association with cerebral aneurysms), even heart failure signs and symptoms in severe cases. Therefore, it is essential to measure the blood pressure in adolescent/young patients in both the upper and lower limbs, as well as to listen in the left subclavian area, where the systolic murmur generated by arterial stenosis can be perceived.

\section{Therapeutic approach to HTN in patients with AOC}

There is currently a unanimous opinion that, in the case of a hemodynamically significant AOC, interventional therapy is recommended ${ }^{13}$. Preoperative pharmacological therapy of HTN in patients with AOC must carefully balance the need to obtain control of blood pressure in the upper part of the body, without endangering mesenteric and renal perfusion. It is recommended to preferably use beta-blockers ${ }^{16}$ and to cautiously use angiotensin receptor blockers or angiotensin converting enzyme inhibitors (these latter drugs should be avoided in very severe cases of AOC, especially if the patient also presents with aortic regurgitation and signs of heart failure). The use of preoperative beta-blockers has been accompanied in some studies by a decrease in the incidence of paroxysmal postoperative $\mathrm{HTN}^{17,18}$.

One of the most common postoperative complications in these patients is persistent HTN. In a study of 404 patients who underwent surgery for aortic coarctation, Heger A et a ${ }^{19}$ showed that most patients had HTN at long-term post-procedural follow-up, whereas only a minority of them showed significant associated restenosis. Immediately, short-term treatment of post-interventional HTN can be performed with i.v. vasodilators (e.g. sodium nitroprusside) or beta-blockers (i.e. esmolol) ${ }^{20}$. If long-term antihypertensive therapy is needed, the drugs of choice are beta-blockers and, depending on the circumstances, angiotensin receptor blockers or angiotensin converting enzyme inhibitors may also be associated.

\section{Aortic Stenosis - PATHOPHYSIOLOGICAL AND THERAPEUTIC IMPLICATIONS}

Historically, patients with aortic stenosis (AS) are thought to rarely present with HTN. It is even considered by some clinicians that the presence of significant HTN theoretically excludes severe AS. Nowadays, echocardiography allows for an early diagnosis of valvular remodeling (due to calcifications of the aortic valve) that occurs in elderly patients and has replaced rheumatic heart disease as the most common cause of aortic stenosis in the developed countries. By combining these two trends, it means that systemic HTN is a more common comorbidity in patients with AS and that it coexists for a longer period before the aortic valve is eventually replaced. In studies involving younger patients with aortic stenosis, the prevalence of HTN is $30 \%$ to $40 \%$, while in three recent case-series involving older patients at increased risk of aortic valve replacement the prevalence of HTN is $75 \%$ or greater ${ }^{21}$.

\section{AS - HTN interrelation}

There have been numerous debates about how the presence of HTN could influence the transvalvular pressure gradient and the assessment of the severity of AS. Theoretically, an increased blood pressure causes an increase in peripheral vascular resistance and a decrease in vascular compliance, leading to changes in transvalvular flow. Clearly, all parameters used for the clinical evaluation of AS are flow-dependent, so they are influenced by the presence of HTN. Numerous in vivo studies have highlighted the influence of blood pressure on the pathophysiology of heart disease in patients with AS, and the influence on ultrasound assessment of the severity of AS, but they also stress out that the way in which HTN influences the AS severity assessment is unpredictable. It is therefore recommended to normalize the blood pressure values before assessing the severity of AS.

Summarizing the current data on the AS-HTN interrelation ${ }^{22}$, we can point out the following aspects: - HTN is an independent risk factor for developing aortic valvular sclerosis and calcification; HTN per se can induce valvular morphological abnormalities, as for every $20 \mathrm{~mm} \mathrm{Hg}$ increase in BP there is a significant increase in the risk of AS and aortic regurgitation ${ }^{22}$. This could be partially explained by the fact that the presence of HTN induces an increased tension on the aortic valves, followed by valvular endothelial lesions ${ }^{23}$.

- Coexistence of HTN with AS implies an additional increase in ventricular afterload, with increased ventricular hypertrophy and remodeling. The presence of HTN-induced myocardial remodeling is associated with a worse prognosis of AS than in the absence of HTN. In clinical practice, the hemodynamic assessment of arterial afterload can be performed by evaluating the valvular-arterial impedance, a parameter that evaluates both the valvular and the vascular load. Increased valvular-aortic impedance has been shown to be associated with worse outcomes, in both symptomatic and asymptomatic patients with moderate or severe $\mathrm{AS}^{24,25}$.

- The association of HTN with AS is considered to play a role in the onset of symptoms of AS, with patients developing symptoms at larger valvular areas than patients without $\mathrm{HTN}^{22}$. 
- Lack of blood pressure control interferes with echocardiographic and cardiac catheterization assessment of the severity of AS, which requires re-evaluation after obtaining $\mathrm{BP}$ control ${ }^{22}$.

- After valve replacement, there may be a higher likelihood for detecting systemic HTN, due to the increased blood flow brought on by the decrease in valvular afterload ${ }^{26}$. Therefore, both before and after valve replacement, treatment of systemic HTN should be an important goal of medical therapy.

\section{Management of patients with AS and HTN}

Given the above considerations, in the management of patients with AS, it is important to recognize the high prevalence of coexisting systemic HTN, its harmful effects on ventricular remodeling and left ventricle function, and the negative impact of valvular replacement therapy on clinical outcomes. Moreover, when determining the severity of AS and making decisions about valve replacement, it is important to recognize that systemic HTN can influence the assessment of the severity of AS (generally causing overestimation of severity).

An analysis of the available clinical data reveals a reluctance of clinicians regarding the treatment of HTN and achievement of the optimal guideline-recommended blood pressure values in patients with AS, despite the fact that the negative prognostic significance of the association of the two conditions is well established. This restraint lies in the recognized fact that fixed valvular obstruction is the main mechanism of afterload increase in patients with AS, and the use of vasodilator therapy could generate an increase in the transvalvular gradient, resulting in arterial vasodilation unaccompanied by a compensatory increase in cardiac output. Current guidelines recommend the treatment of HTN in patients with AS, emphasizing that they should be carefully monitored, with more frequent follow-up visits, and the treatment must be titrated accordingly $y^{27,28}$. To date, there are no prospective randomized studies recommending the use of a particular class of antihypertensive drugs in patients with AS.

The use of medication, such as angiotensin converting enzyme inhibitors and angiotensin II receptor blockers, has been associated with an improvement in certain "hard" endpoints, such as mortality and cardiovascular events ${ }^{29}$. Moreover, there are no published studies or reported cases to date that establish a clear link between the use of angiotensin converting enzyme inhibitors or angiotensin II receptor blockers and symptomatic hypotension in patients with AS. In the SCOPE-AS study, Chockalingam et $\mathrm{al}^{30}$ reported a good tolerance of angiotensin converting enzyme inhibitors therapy, even in symptomatic patients with severe AS who were not eligible candidates for valvular replacement therapy, demonstrating a preservation of the left ventricular ejection fraction. Following valve replacement therapy, the use of an angiotensin II receptor blocker (candesartan) has been associated with a more significant regression of ventricular remodeling compared to patients treated with standard medical therapy ${ }^{31}$. In summary, angiotensin converting enzyme inhibitors or angiotensin II receptor blockers therapy is not only a viable therapeutic alternative in patients with AS, but it also has a positive impact on patient's outcome; nonetheless, it requires careful initiation and titration of doses, especially in patients with severe AS and low ejection fraction.

Treatment with calcium channel blockers seems to be associated with a decrease in survival, so it is not to be chosen as the first therapeutic line. Diuretic drugs should be used with caution and only in patients with clinical features suggestive of systemic or pulmonary congestion. Excessive decrease in preload can cause a decrease in ventricular filling and can lead to a decrease in heart rate and blood pressure (even more in patients with myocardial remodeling and heart failure with reduced ejection fraction). One should especially avoid diuretic therapy as much as possible in patients with severe secondary left ventricular hypertrophy with a small ventricular volume.

The use of beta-blockers in patients with AS may be indicated especially in patients who associate ischemic coronary heart disease, as it causes a decrease in the heart rate and aortic ejection velocity and may thus reduce the hemodynamic stress. If these assumptions have been shown to be consistent for patients with severe-to-moderate AS, the use of beta-blockers in patients with tight AS should be done with caution and the target heart rate should be higher than in patients without AS.

It is important to emphasize the high prevalence of the coexistence of AS and HTN and to acknowledge the fact that HTN is an additional negative prognostic factor in these patients, thus requiring effective antihypertensive therapy ${ }^{32}$. Sahrai et $\mathrm{al}^{33}$ recently pointed out in an analysis that all data come from observational studies and there are no recommendations on the class of antihypertensive drugs recommended as first-line treatment in these patients, but evidence from studies proves the beneficial effect on ventricular remodeling of RAAS inhibitors ${ }^{29,30}$. Regardless of the therapeutic options chosen, the need for repeated medication titration and frequent reassessment should be emphasized, especially in patients with severe AS with or without reduction of the left ventricle ejection fraction. In the management of patients with symptomatic severe AS and uncontrolled HTN, the reassessment of the severity of AS after achieving optimal 
control of the blood pressure should be considered, especially in borderline cases between moderate and severe AS. The suggested therapeutic blood pressure target is $130-139 \mathrm{mmHg}$ for systolic blood pressure and $70-90 \mathrm{mmHg}$ for diastolic blood pressure, and lower values should be avoided 27,28 .

\section{Chronic aortic regurgitation - pathophysio- LOGICAL AND THERAPEUTIC IMPLICATIONS}

The relationship between chronic aortic regurgitation and HTN is reciprocal, in the sense that aortic regurgitation can generate HTN and this in turn can influence the natural evolution of the valvular insufficiency.

Chronic aortic regurgitation causes a consequent increase in the end-diastolic left ventricle volume, accompanied by a lower increase in the end-systolic left ventricle volume. Long-term changes include the dilation of the left ventricle cavity, with an increase of the systolic transvalvular flow and the increase of the systolic blood pressure, tachycardia, producing a characteristic compensatory hyperkinetic syndrome. Diastolic blood pressure will be decreased due to regurgitation of blood in the left ventricle, leading to a wide-pulse pressure HTN. The appearance of this characteristic pattern of blood pressure is a sign of severity of the valvular disease (more specific a sign of an important regurgitated volume).

The coexistence of HTN with aortic regurgitation has a deleterious pathophysiological effect on the cardiac adaptive processes. On the one hand, it increases the afterload (due to the increase in blood pressure), increasing the volume of blood regurgitated during diastole, while on the other hand the arterial wall develops typical HTN changes (hyalinization, destruction of elastin fibres) with important central consequences. At the same time with the development of the left ventricular hypertrophy, typically seen in hypertensive patients, a diastolic dysfunction of the heart appears, ultimately leading to a secondary increase in the myocardial wall stress, and thus inducing left ventricle dilation in the long-term.

Considering these pathophysiological aspects and the animal studies that found that mice with HTN and aortic regurgitation had a more severe left ventricle hypertrophy, the use of vasodilator treatment in patients with chronic severe aortic regurgitation was proposed. The American College of Cardiology/American Heart Association guidelines ${ }^{27}$ currently have the following recommendations for vasodilator therapy in patients with chronic aortic regurgitation:

- ong-term vasodilatory therapy is recommended for patients with severe chronic aortic regurgitation and symptoms of left ventricle dysfunction, with or without HTN;

- vasodilatory therapy is reasonable in patients with asymptomatic severe aortic regurgitation with left ventricle dilation and preserved ejection fraction, regardless of whether they also have HTN;

- vasodilatory therapy is not recommended for asymptomatic patients with non-severe aortic regurgitation and without left ventricle dysfunction unless they also have HTN.

\section{Conclusions}

The coexistence of arterial hypertension and aortic diseases is far to be a rarity. Clinicians should be proactive in searching for aortic aneurysms or aortic valvular diseases in hypertensive patients. The association of arterial hypertension with these pathological conditions puts peculiar challenges in the treatment and monitoring of the patients.

\section{Author Contributions:}

C.M.T. conceived the original draft preparation, was responsible for the data acquisition, collection and assem. bly of the articles/published data, and their inclusion and interpretation in this review.

\section{Compliance with Ethics Requirements:}

„The authors declare no conflict of interest regarding this article"

„No funding for this study“

\section{Acknowledgements: \\ None}

\section{References}

1. Dorobantu M, Darabont R, Dimulescu D, et al. New epidemiologic national representative survey; SEPHAR III - main results. Journal of Hypertension. 2017(35):e60.

2. $2018 \mathrm{ESC} / \mathrm{ESH}$ Guidelines for the management of arterial hypertension: The Task Force for the management of arterial hypertension of the European Society of Cardiology (ESC) and the European Society of Hypertension (ESH). European Heart Journal. 2018;39(33):3021-3104.

3. Tomiyama H, Matsumoto C, Shiina K, Yamashina A. Brachial-ankle PWV: current status and future directions as a useful marker in the management of cardiovascular disease and/or cardiovascular risk factors. J Atheroscler Thromb. 2016;23(2):128-46

4. Mitchell GF, Hwang S-J, Vasan RS, et al Arterial stiffness and cardiovascular events: the Framingham Heart Study. Circulation. 2010;121(4):505-11.

5. Mitchell GF. Arterial stiffness and hypertension - chicken or egg? Hypertension. 2014;64:210-214 
6. Williams B. Evaluating interventions to reduce central aortic pressure, arterial stiffness and morbidity-mortality. J Hypertens. 2012;30(Suppl):S13-S18.

7. Shahin Y, Khan JA, Chetter I. Angiotensin converting enzyme inhibitors effect on arterial stiffness and wave reflections: a meta-analysis and meta-regression of randomised controlled trials. Atherosclerosis. 2012;221:18-33.

8. Gianfagna F, Veronesi G, Bertù L, et al. Prevalence of abdominal aortic aneurysms and its relation with cardiovascular risk stratification: protocol of the risk of cardiovascular diseases and abdominal aortic aneurysm in Varese (RoCAV) population based study. BMC Cardiovasc Disord. 2016; 16: 243.

9. Braverman AC. Acute aortic dissection. Circulation. 2010; 122(2):184-188.

10. Milan A, Tosello F, Naso D, et al. Ascending aortic dilatation, arterial stiffness and cardiac organ damage in essential hypertension. Journal of Hypertension. 2013;31(1):109_ 116.

11. Crawford ES, Salwa SA, Babb JW III, Glaeser DH, Vaccaro PS, Silvers A. Infrarenal abdominal aortic aneurysm. Factors influencing survival after operation performed over a 25 year period. Ann Surg 1981;193:699-709.

12. https://www.escardio.org/Guidelines/Clinical-PracticeGuidelines/Aortic-Diseases (accessed 02 Nov 2020)

13. Ginghina C. Mic tratat de cardiologie. Editura Academiei Romane, Bucuresti, 2010, pg 575-577.

14. Scott HW Jr, Bahnson HT. Evidence for a renal factor in the hypertension of experimental coarctation of the aorta. Surgery. 1951;30(1):206-17.

15. Scott HW Jr, Collins HA, Langa AM, Olsen NS. Additional observations concerning the physiology of the hypertension associated with experimental coarctation of the aorta. Surgery. 1954:36(3):445-59.

16. Lopez-Sendon J, Swedberg K, McMurray J, et al. Expert consensus document on beta-adrenergic receptor blockers. Eur Heart J. 2004;25(15):1341-62.

17. Sealy WC, Harris JS, Young WG Jr, Callaway HA Jr. Paradoxical hypertension following resection of coarctation of aorta. Surgery. 1957;42(1):135-47.

18. Tabbutt S, Nicolson SC, Adamson PC, Zhang X, Hoffman ML, Wells W. The safety, efficacy, and pharmacokinetics of esmolol for blood pressure control immediately after repair of coarctation of the aorta in infants and children: a multicenter, double-blind, randomized trial. J Thorac Cardiovasc Surg. 2008;136(2):321-8.

19. Hager A, Kanz S, Kaemmerer H, et al. Coarctation Long-term Assessment (COALA): significance of arterial hypertension in a cohort of 404 patients up to 27 years after surgical repair of isolated coarctation of the aorta, even in the absence of restenosis and prosthetic material. The Journal of Thoracic and Cardiovascular Surgery. 2007;134(3):738-745.

20. http://www. cvphysiology.com/Heart\%20Disease/ HD009b.htm (accessed 03 Nov 2020)
21. Stewart BF, Siscovick D, Lind BK, et al. Clinical factors associated with calcific aortic valve disease. Cardiovascular Health Study. J Am Coll Cardiol. 197;29(3):630-634.

22. Manolis AJ, Kallistratos MS, Poulimenos LE. Hypertension and aortic stenosis - no strangers, not anymore! Journal of Hypertension. 2019; 37(11):2156-2158.

23. Cuniberti LA, Stutzbach PG, Guevara E, Yannarelli GG, Laguens RP, Favaloro RR. Development of mild aortic valve stenosis in a rabbit model of hypertension. J Am Coll Cardiol. 2006;47(11):2303-2309.

24. Hachicha Z, Dumesnil JG, Pibarot P. Usefulness of the valvuloarterial impedance to predict adverse outcome in asymptomatic aortic stenosis. J Am Coll Cardiol. 2009;54(11):10031011.

25. Lancellotti P, Donal E, Magne J et al. Risk stratification in asymptomatic moderate to severe aortic stenosis: the importance of the valvular, arterial and ventricular interplay. Heart. 2010; 96(17):1364-1371.

26. Rodés-Cabau J, Webb JG, Cheung A, et al. Transcatheter aortic valve implantation for the treatment of severe symptomatic aortic stenosis in patients at very high or prohibitive surgical risk: acute and late outcomes of the multicenter Canadian experience. J Am Coll Cardiol. 2010;55(11):10801090.

27. Nishimura RA, Otto CM, Bonow RO, et al. American College of Cardiology/American Heart Association Task Force on Practice Guidelines. 2014 AHA/ACC guideline for the management of patients with valvular heart disease: executive summary: a report of the American College of Cardiology/ American Heart Association Task Force on Practice Guidelines. J Am Coll Cardiol. 2014;63(22):24382488.

28. Vahanian A, Alfieri O, Andreotti F, et al. Guidelines on the management of valvular heart disease (version 2012): the Joint Task Force on the Management of Valvular Heart Disease of the European Society of Cardiology (ESC) and the European Association for Cardio-Thoracic Surgery (EACTS). Eur Heart J. 2012;33(19):2451-2496.

29. Shavelle DM. Are angiotensin converting enzyme inhibitors beneficial in patients with aortic stenosis? Heart. 2005;91(10):1257-1259.

30. Chockalingam A, Venkatesan S, Subramaniam T, et al. Safety and efficacy of angiotensin converting enzyme inhibitors in symptomatic severe aortic stenosis: Symptomatic Cardiac Obstruction-Pilot Study of Enalapril in Aortic Stenosis (SCOPE-AS). Am Heart J. 2004;147(4): E19.

31. Dahl JS, Videbaek L, Poulsen MK, et al. Effect of candesartan treatment on left ventricular remodeling after aortic valve replacement for aortic stenosis. Am J Cardiol. 2010;106(5):713-719.

32. Lindman BR, Otto CM. Time to treat hypertension in patients with aortic stenosis. Circulation. 2013;128:1281-1283.

33. Saeed S, Scalise F, Chambers JB, et al. Hypertension in aortic stenosis: a focused review and recommendations for clinical practice. Journal of Hypertension. 2020;38(7):1211-1219 